\title{
The Turán problem for hypergraphs of fixed size
}

\author{
Peter Keevash \\ Department of Mathematics \\ Caltech, Pasadena, CA 91125, USA. \\ keevash@caltech.edu \\ Submitted: Oct 22, 2004; Accepted: Jun 3, 2005; Published: Jun 14, 2005 \\ Mathematics Subject Classifications: 05 D05
}

\begin{abstract}
We obtain a general bound on the Turán density of a hypergraph in terms of the number of edges that it contains. If $\mathcal{F}$ is an $r$-uniform hypergraph with $f$ edges we show that $\pi(\mathcal{F})<\frac{f-2}{f-1}-(1+o(1))\left(2 r !^{2 / r} f^{3-2 / r}\right)^{-1}$, for fixed $r \geq 3$ and $f \rightarrow \infty$.
\end{abstract}

Given an $r$-uniform hypergraph $\mathcal{F}$, the Turán number of $\mathcal{F}$ is the maximum number of edges in an $r$-uniform hypergraph on $n$ vertices that does not contain a copy of $\mathcal{F}$. We denote this number by $\operatorname{ex}(n, \mathcal{F})$. It is not hard to show that the limit $\pi(\mathcal{F})=$ $\lim _{n \rightarrow \infty} e x(n, \mathcal{F}) /\left(\begin{array}{l}n \\ r\end{array}\right)$ exists. It is usually called the Turán density of $\mathcal{F}$. There are very few hypergraphs with $r>2$ for which the Turán density is known, and even fewer for the exact Turán number. We refer the reader to $[10,11,12,13,14,15,16]$ for recent results on these problems.

A general upper bound on Turán densities was obtained by de Caen [3], who showed $\pi\left(K_{s}^{(r)}\right) \leq 1-\left(\begin{array}{c}s-1 \\ r-1\end{array}\right)^{-1}$, where $K_{s}^{(r)}$ denotes the complete $r$-uniform hypergraph on $s$ vertices. A construction showing $\pi\left(K_{s}^{(r)}\right) \geq 1-\left(\frac{r-1}{s-1}\right)^{r-1}$ was given by Sidorenko [17] (see also [18]); better bounds are known for large $r$. We refer the reader to Sidorenko [18] for a full discussion of this problem. For a general hypergraph $\mathcal{F}$ Sidorenko [19] (see also [20]) obtained a bound for the Turán density in terms of the number of edges, showing that if $\mathcal{F}$ has $f$ edges then $\pi(\mathcal{F}) \leq \frac{f-2}{f-1}$. In this note we improve this as follows.

Theorem 1 Suppose $\mathcal{F}$ is an $r$-uniform hypergraph with $f$ edges.

(i) If $r=3$ and $f \geq 4$ then $\pi(\mathcal{F}) \leq \frac{1}{2}\left(\sqrt{f^{2}-2 f-3}-f+3\right)$.

(ii) For a fixed $r \geq 3$ and $f \rightarrow \infty$ we have $\pi(\mathcal{F})<\frac{f-2}{f-1}-(1+o(1))\left(2 r !^{2 / r} f^{3-2 / r}\right)^{-1}$.

We start by describing our main tool, which is Sidorenko's analytic approach. See [20] for a survey of this method. Consider an $r$-uniform hypergraph $\mathcal{H}$ on $n$ vertices. It is convenient to regard the vertex set $V$ as a finite measure space, in which each vertex $v$ has $\mu(\{v\})=1 / n$, so that $\mu(V)=1$. We write $h: V^{r} \rightarrow\{0,1\}$ for the symmetric function 
$h\left(x_{1}, \cdots, x_{r}\right)$ which takes the value 1 if $\left\{x_{1}, \cdots, x_{r}\right\}$ is an edge of $\mathcal{H}$ and 0 otherwise. Then $\int h d \mu^{r}=r ! e(\mathcal{H}) n^{-r}=d+O(1 / n)$, where $d=\left(\begin{array}{l}n \\ r\end{array}\right)^{-1} e(\mathcal{H})$ is the density of $\mathcal{H}$.

Now consider a fixed forbidden $r$-uniform hypergraph $\mathcal{F}$ with $f$ edges on the vertex set $\{1, \cdots, m\}$. We associate to vertex $i$ the variable $x_{i}$, and to an edge $e=\left\{i_{1}, \cdots, i_{r}\right\}$ the function $h_{e}(x)=h\left(x_{i_{1}}, \cdots, x_{i_{r}}\right)$, where $x$ denotes the vector $\left(x_{1}, \cdots, x_{m}\right)$. The configuration product of $\mathcal{F}$ with respect to $h$ is the function $h_{\mathcal{F}}(x)=\prod_{e \in \mathcal{F}} h_{e}(x)$. Then

$\int h_{\mathcal{F}} d \mu^{m}=n^{-m} \operatorname{hom}(\mathcal{F}, \mathcal{H})=n^{-m} \operatorname{mon}(\mathcal{F}, \mathcal{H})+O\left(n^{-1}\right)=n^{-m} \operatorname{aut}(\mathcal{F}) \operatorname{sub}(\mathcal{F}, \mathcal{H})+O\left(n^{-1}\right)$,

where $\operatorname{hom}(\mathcal{F}, \mathcal{H})$ is the number of homomorphisms (edge-preserving maps) from $\mathcal{F}$ to $\mathcal{H}$, $\operatorname{mon}(\mathcal{F}, \mathcal{H})$ is the number of these that are monomorphisms (injective homomorphisms), $\operatorname{aut}(\mathcal{F})$ is the number of automorphisms of $\mathcal{F}$ and $\operatorname{sub}(\mathcal{F}, \mathcal{H})$ is the number of $\mathcal{F}$-subgraphs of $\mathcal{H}$. Also, Erdős-Simonovits supersaturation [6] implies that for any $\delta>0$ there is $\epsilon>0$ and an integer $n_{0}$ so that for any $r$-uniform hypergraph $\mathcal{H}$ on $n \geq n_{0}$ vertices with $\left(\begin{array}{l}n \\ r\end{array}\right)^{-1} e(\mathcal{H})>\pi(\mathcal{F})+\delta$ we have $n^{-m} \operatorname{sub}(\mathcal{F}, \mathcal{H})>\epsilon$. It follows that

$$
\pi(\mathcal{F})=\inf _{\epsilon>0} \liminf _{|V| \rightarrow \infty} \max _{h: V^{r} \rightarrow\{0,1\}, \int h_{\mathcal{F}} d \mu^{m}<\epsilon} \int h d \mu^{r} .
$$

We say that $\mathcal{F}$ is a forest if we can order its edges as $e_{1}, \cdots, e_{f}$ so that for every $2 \leq i \leq f$ there is some $1 \leq j \leq i-1$ so that $e_{i} \cap\left(\cup_{t=1}^{i-1} e_{t}\right) \subset e_{j}$. Sidorenko [20] showed that if $\mathcal{F}$ is a forest with $f$ edges then

$$
\int h_{\mathcal{F}} d \mu^{m} \geq\left(\int h d \mu^{r}\right)^{f}
$$

Now we need a lemma on when a hypergraph contains a forest of given size.

Lemma 2 (i) An r-uniform hypergraph with at least $r !(t-1)^{r}$ edges contains a forest with $t$ edges.

(ii) Let $\mathcal{F}$ be a 3-uniform hypergraph. Then either (a) $\mathcal{F}$ contains a forest with 3 edges, or (b) $\pi(\mathcal{F})=0$, or $(c) \mathcal{F} \subset K_{4}^{(3)}$, or (d) $\mathcal{F}=\mathcal{F}_{5}=\{a b c, a b d, c d e\}$.

Proof. (i) This is immediate from the result of Erdös and Rado [5] that such a hypergraph contains a sunflower with $t$ petals, i.e. edges $e_{1}, \cdots, e_{t}$ for which all the pairwise intersections $e_{i} \cap e_{j}$ are equal. A sunflower is in particular a forest.

(ii) Consider a 3 -uniform hypergraph $\mathcal{F}$ that does not contain a forest with 3 edges. We can assume that $\mathcal{F}$ is not 3-partite (Erdős [4] showed that this implies $\pi(\mathcal{F})=0$ ) so $\mathcal{F}$ has at least 3 edges. Clearly $\mathcal{F}$ cannot have two disjoint edges, as then adding any other edge gives a forest.

Suppose there is a pair of edges that share two points, say $e_{1}=a b c$ and $e_{2}=a b d$. Any other edge must contain $c$ and $d$, or together with $e_{1}$ and $e_{2}$ we have a forest. Consider another edge $e_{3}=c d e$. If there are no other edges then either $\mathcal{F}=\mathcal{F}_{5}$ or $\mathcal{F} \subset K_{4}^{(3)}$ (if $e$ 
equals $a$ or $b$ ). If there is another edge $e_{4}=c d f$ then the same argument shows that $e_{1}$ and $e_{2}$ both contain $e$ and $f$, i.e. $\mathcal{F}=K_{4}^{(3)}$ and there can be no more edges.

The other possibility is that every pair of edges intersect in exactly one point. Then there are at most 2 edges containing any point, or we would have a forest with 3 edges. Consider three edges, which must have the form $e_{1}=a b c, e_{2}=c d e, e_{3}=e f a$. There can be at most one more edge $e_{4}=b d f$. But this forms a 3-partite hypergraph (with parts $a d, b e, c f)$, a case we have already excluded. This proves the lemma.

Proof of Theorem. Let $\mathcal{F}$ be an $r$-uniform hypergraph with $f$ edges that contains a forest $\mathcal{T}$ with $t$ edges. Label the edges $e_{1}, \cdots, e_{f}$, where $e_{1}, \cdots, e_{t}$ are the edges of $\mathcal{T}$. Suppose that $\mathcal{H}$ is an $r$-uniform hypergraph on a vertex set $V$ of size $n$. Define the measure $\mu$ and the function $h: V^{r} \rightarrow\{0,1\}$ as before. Observe the inequality

$$
h_{\mathcal{F}}(x) \geq h_{\mathcal{T}}(x)+\sum_{i=t+1}^{f} h_{e_{1}}(x)\left(h_{e_{i}}(x)-1\right)
$$

This holds, as the second term is non-positive (since $h_{e}(x) \in\{0,1\}$ ), so it could only fail for some $x$ if $h_{\mathcal{F}}(x)=0$ and $h_{\mathcal{T}}(x)=1$. But then we have $h_{e_{1}}(x)=\cdots=h_{e_{t}}(x)=1$ and $h_{e_{i}}(x)=0$ for some $i>t$, and the term $h_{e_{1}}(x)\left(h_{e_{i}}(x)-1\right)=-1$ cancels $h_{\mathcal{T}}(x)$, so the inequality holds for all $x$. Integrating gives

$$
\int h_{\mathcal{F}}(x) d \mu^{m} \geq \int h_{\mathcal{T}}(x) d \mu^{m}+\sum_{i=t+1}^{f} \int h_{e_{1}}(x) h_{e_{i}}(x)-h_{e_{1}}(x) d \mu^{m} \geq p^{t}+(f-t)\left(p^{2}-p\right),
$$

where we write $p=\int h d \mu^{r}$ and apply the inequality (2) for the forests $\mathcal{T}$ and $\left\{e_{1}, e_{i}\right\}$, $t+1 \leq i \leq f$. By equation (1) we deduce that the Turán density $\pi=\pi(\mathcal{F})$ satisfies $\pi^{t}+(f-t)\left(\pi^{2}-\pi\right) \leq 0$.

Writing $g(x)=x^{t-1}+(f-t)(x-1)$ we either have $\pi=0$ or $g(\pi) \leq 0$. Now $g(0)=-(f-t) \leq 0, g(1)=1$ and $\frac{d g}{d x}=(t-1) x^{t-2}+f-t \geq 0$ for $0<x<1$ so $g$ has exactly one root $\alpha$ in $[0,1]$, and $\pi \leq \alpha$.

First we consider the case $r=3$. If $f \geq 5$ then by the lemma we can take $t=3$. Solving the quadratic $g(x)=x^{2}+(f-3)(x-1)=0$ gives $\pi \leq \alpha=\frac{1}{2}\left(\sqrt{f^{2}-2 f-3}-f+3\right)$. This also holds when $f=4$, as then by the lemma we may suppose that $\mathcal{F}=K_{4}^{(3)}$. Chung and $\mathrm{Lu}[2]$ showed that $\pi\left(K_{4}^{(3)}\right) \leq \frac{3+\sqrt{17}}{12}$ which is less than $\frac{1}{2}(\sqrt{5}-1)$.

Now consider the case when $r \geq 3$ is fixed and $f \rightarrow \infty$. By the lemma we can take $t=$ $(f / r !)^{1 / r}$. Write $\alpha=1-\epsilon$. Since $g(\alpha)=0$ we have $(f-t) \epsilon=(1-\epsilon)^{t-1}<1$, so $\epsilon<1 /(f-t)$. From the Taylor expansion of $(1-\epsilon)^{t-1}$ we have $(f-t) \epsilon>1-(t-1) \epsilon+\left(\begin{array}{c}t-1 \\ 2\end{array}\right) \epsilon^{2}-\left(\begin{array}{c}t-1 \\ 3\end{array}\right) \epsilon^{3}$. Also $\left(\begin{array}{c}t-1 \\ 3\end{array}\right) \epsilon^{3}<\frac{1}{6}\left(\frac{t-1}{f-t}\right)^{3}<\frac{1}{6}(t / f)^{3}$ (since $\left.f>t^{2}\right)$ so $\left(\begin{array}{c}t-1 \\ 2\end{array}\right) \epsilon^{2}-(f-1) \epsilon+1-\frac{1}{6}(t / f)^{3}<0$. 
Writing $\Delta=(f-1)^{2}-4\left(\begin{array}{c}t-1 \\ 2\end{array}\right)\left(1-\frac{1}{6}(t / f)^{3}\right)$ for the discriminant of this quadratic we have

$$
\begin{aligned}
\epsilon & >\frac{f-1-\Delta^{1 / 2}}{(t-1)(t-2)}=\frac{2\left(1-\frac{1}{6}(t / f)^{3}\right)}{f-1+\Delta^{1 / 2}} \\
& =\frac{2}{f-1}\left(1+\left(1-2(t-1)(t-2)\left(1-\frac{1}{6}(t / f)^{3}\right)(f-1)^{-2}\right)^{1 / 2}\right)^{-1}+O\left(t^{3} / f^{4}\right) \\
& =\frac{2}{f-1}\left(1+1-(t-1)(t-2)(f-1)^{-2}+O\left(t^{4} / f^{4}\right)\right)^{-1}+O\left(t^{3} / f^{4}\right) \\
& =\frac{1}{f-1}\left(1+\frac{1}{2}(t-1)(t-2)(f-1)^{-2}+O\left(t^{4} / f^{4}\right)\right)+O\left(t^{3} / f^{4}\right) \\
& =\frac{1}{f-1}+\frac{(t-1)(t-2)}{2(f-1)^{3}}+O\left(t^{3} / f^{4}\right) .
\end{aligned}
$$

Since $\alpha=1-\epsilon$ and $t=(f / r !)^{1 / r}$ we have

$$
\pi \leq \alpha<\frac{f-2}{f-1}-(1+o(1))\left(2 r !^{2 / r} f^{3-2 / r}\right)^{-1} .
$$

This proves the theorem.

Remarks. (1) For a graph $G$ we have $e(G) \geq\left(\begin{array}{c}\chi(G) \\ 2\end{array}\right)$ with equality if and only if $G$ is complete. The Erdős-Stone theorem [7] implies that $\pi(G)=\frac{\chi(G)-2}{\chi(G)-1}<1-\frac{1+o(1)}{\sqrt{2 e(G)}}$. It is natural to think that complete hypergraphs should also have the highest Turán density among all hypergraphs with the same number of edges. Were this true de Caen's bound would give $\pi(\mathcal{F})<1-\Omega\left(f^{-(r-1) / r}\right)$ for an $r$-uniform hypergraph $\mathcal{F}$ with $f$ edges.

(2) If $\mathcal{F}$ has 3 edges then Sidorenko's bound $\pi(\mathcal{F}) \leq 1 / 2$ is tight when $\mathcal{F}=K_{3}^{(2)}$ is a triangle, or more generally when $\mathcal{F}$ is the $2 k$-uniform hypergraph with edges $\left\{P_{1} \cup\right.$ $\left.P_{2}, P_{2} \cup P_{3}, P_{3} \cup P_{1}\right\}$, where $P_{1}, P_{2}, P_{3}$ are disjoint sets of size $k$ (see $[8,14]$ ). If $\mathcal{F}$ is 3-uniform and has 3 edges then the lemma shows that $\pi(\mathcal{F}) \leq \max \left\{\pi\left(\mathcal{F}_{4}\right), \pi\left(\mathcal{F}_{5}\right)\right\}$, where $\mathcal{F}_{4}$ denotes the 3-edge subgraph of $K_{4}^{(3)}$ and $\mathcal{F}_{5}=\{a b c, a b d, c d e\}$. Frankl and Füredi [9] showed that $\pi\left(\mathcal{F}_{5}\right)=2 / 9$ and Mubayi [15] showed $\pi\left(\mathcal{F}_{4}\right)<1 / 3-10^{-6}$, so we see that $\pi(\mathcal{F})<1 / 3-10^{-6}$, and Sidorenko's bound is not tight. It would be interesting to determine if it is ever tight for a hypergraph with edges of odd size.

(3) How many edges in an $r$-uniform hypergraph guarantee a forest with $t$ edges? An answer to this question may lead to an improvement in our theorem, and it also seems interesting in its own right. Erdős and Rado [5] conjectured that for any $t$ there is a constant $C$ so that any $r$-uniform hypergraph with $C^{r}$ edges contains a sunflower with $t$ edges. We can obtain a bound of this form for forests, indeed, we claim that any $r$-uniform hypergraph $\mathcal{F}$ with $\left(2^{t}\right)^{r}$ edges contains a forest with $t$ edges. For if we fix any edge $e$, then the other edges have $2^{r}$ possible intersections with it, so we can find a hypergraph $\mathcal{F}^{\prime} \subset \mathcal{F} \backslash e$ with $\left(2^{t-1}\right)^{r}$ edges, all of which have the same intersection with $e$. By induction we can find a forest with $t-1$ edges in $\mathcal{F}^{\prime}$, and adding $e$ gives a forest of size $t$ in $\mathcal{F}$. 
Actually, it is not hard to improve this bound to $2\left(\begin{array}{c}r \\ r / 2\end{array}\right)^{t-2}$. For we only need the intersections $\left\{e \cap e^{\prime}: e^{\prime} \in \mathcal{F}\right\}$ to form a chain, and the subsets of $e$ can be partitioned into $\left(\begin{array}{c}r \\ r / 2\end{array}\right)$ chains (see, for example, [1] page 10). Thus we need only lose a factor $\left(\begin{array}{c}r \\ r / 2\end{array}\right)$ at each induction step, and after $t-2$ steps we get down to a 2-edge forest.

However, this bound does not help in our application, as we are interested in the case when $r$ is fixed and $t$ is large. We have an upper bound of $r ! t^{r}$ from Erdös and Rado, and and noting that $K_{r+t-2}^{(r)}$ does not contain a forest with $t$ edges we obtain a lower bound of $\left(\begin{array}{c}r+t-2 \\ r\end{array}\right) \sim t^{r} / r$ !, so we have a constant $r !^{2}$ factor of uncertainty.

\section{References}

[1] B. Bollobás, Combinatorics, Set systems, hypergraphs, families of vectors and combinatorial probability, Cambridge University Press, Cambridge, 1986.

[2] F. Chung and L. Lu, An upper bound for the Turán number $t_{3}(n, 4)$, J. Combin. Theory Ser. A 87 (1999), 381-389.

[3] D. de Caen, Extension of a theorem of Moon and Moser on complete subgraphs, Ars Combin. 16 (1983), 5-10.

[4] P. Erdős, On extremal problems of graphs and generalized graphs, Israel J. Math. 2 (1964), 183-190.

[5] P. Erdös and R. Rado, Intersection theorems for systems of sets, J. London Math. Soc. 35 1960, 85-90.

[6] P. Erdős and M. Simonovits, Supersaturated graphs and hypergraphs, Combinatorica 3 (1983), 181-192.

[7] P. Erdős and A. H. Stone, On the structure of linear graphs, Bull. Amer. Math. Soc. 52 (1946), 1087-1091.

[8] P. Frankl, Asymptotic solution of a Turán-type problem. Graphs and Combinatorics 6 (1990), 223-227.

[9] P. Frankl, Z. Füredi, A new generalization of the Erdős-Ko-Rado theorem, Combinatorica 3 (1983), 341-349.

[10] Z. Füredi, O. Pikhurko and M. Simonovits, On triple systems with independent neighborhoods, Combin. Probab. Comput., to appear.

[11] Z. Füredi and M. Simonovits, Triple systems not containing a Fano Configuration, Combin. Probab. Comput., to appear.

[12] P. Keevash, The Turán problem for projective geometries, J. Combin. Theory Ser. A, to appear. 
[13] P. Keevash and B. Sudakov, The exact Turán number of the Fano plane, Combinatorica, to appear.

[14] P. Keevash and B. Sudakov, On a hypergraph Turán problem of Frankl, Combinatorica, to appear.

[15] D. Mubayi, On hypergraphs with every four points spanning at most two triples, Electron. J. Combin. 10 (2003), Note 10, 4 pp. (electronic).

[16] D. Mubayi and V. Rödl, On the Turán number of Triple Systems, J. Comb. Theory Ser. A, 100 (2002), 136-152.

[17] A. F. Sidorenko, Systems of sets that have the T-property, Vestnik Moskov. Univ. Ser. I Mat. Mekh. 1981, 19-22.

[18] A. F. Sidorenko, What we know and what we do not know about Turán numbers, Graphs Combin. 11 (1995), 179-199.

[19] A. F. Sidorenko, Extremal combinatorial problems in spaces with continuous measure, Issled. Operatsiü ASU 34 (1989), 34-40.

[20] A. F. Sidorenko, An analytic approach to extremal problems for graphs and hypergraphs, Extremal problems for finite sets (Visegrád, 1991), 423-455, Bolyai Soc. Math. Stud., 3, János Bolyai Math. Soc., Budapest, 1994. 